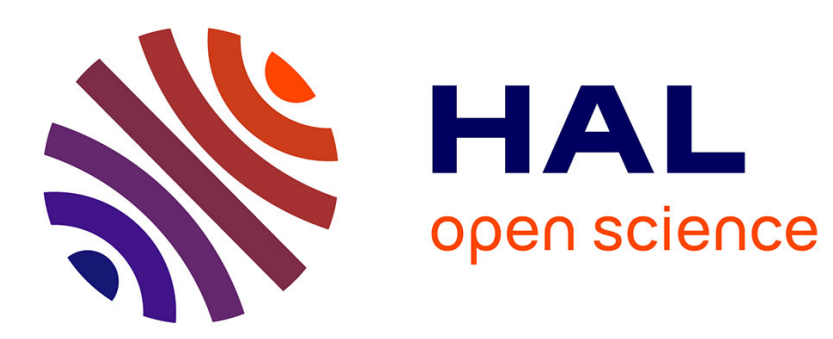

\title{
Experimental Evolution as a Tool to Investigate Natural Processes and Molecular Functions
}

Philippe Remigi, Catherine Masson-Boivin, Eduardo P C Rocha

\section{To cite this version:}

Philippe Remigi, Catherine Masson-Boivin, Eduardo P C Rocha. Experimental Evolution as a Tool to Investigate Natural Processes and Molecular Functions. Trends in Microbiology, 2019, 27 (7), pp.623-634. 10.1016/j.tim.2019.02.003 . hal-02329724

\section{HAL Id: hal-02329724 \\ https://hal.science/hal-02329724}

Submitted on 23 Oct 2019

HAL is a multi-disciplinary open access archive for the deposit and dissemination of scientific research documents, whether they are published or not. The documents may come from teaching and research institutions in France or abroad, or from public or private research centers.
L'archive ouverte pluridisciplinaire HAL, est destinée au dépôt et à la diffusion de documents scientifiques de niveau recherche, publiés ou non, émanant des établissements d'enseignement et de recherche français ou étrangers, des laboratoires publics ou privés. 
3 Philippe Remigi ${ }^{1 *}$, Catherine Masson-Boivin ${ }^{1}$ and Eduardo P.C. Rocha ${ }^{2,3}$

4 1. Laboratoire des Interactions Plantes-Microorganismes (LIPM), Université de Toulouse, 5 INRA, CNRS, 31326 Castanet-Tolosan, France.

6 2. Microbial Evolutionary Genomics, Institut Pasteur, 25-28 rue Dr. Roux, 75015 Paris, France.

7 3. CNRS, UMR3525, 25-28 rue Dr. Roux, 75015 Paris, France

$8 \quad$ *Correspondance: philippe.remigi@inra.fr (P. Remigi)

11 Keywords: Experimental evolution, gene regulation, antibiotic resistance, host-microbe 12 interactions.

\section{Abstract}

Experimental evolution of microbes has allowed evolutionary biologists to examine adaptive processes in real time, generating novel insights into fundamental laws of evolution. Much less appreciated is the potential of this approach to advance the understanding of microbial cells and molecular processes in complement of traditional molecular genetics. The tracking of mutations underlying phenotypic changes offers the opportunity for detailed molecular analyses of novel phenotypes. This provides a breadth of information on diverse biological systems and may retrace key past events of natural history. Here, we highlight how the field has advanced our understanding of gene regulation, antibiotic resistance and hostmicrobiome interactions to exemplify how experimental evolution can be employed to provide new light on microbial systems. 


\section{Highlights}

- Experimental evolution (EE) can complement traditional molecular genetic studies on microbial systems.

- The diversity of EE approaches enabled progresses in many fields of microbiology, including molecular mechanisms of gene regulation, antibiotic resistance, and hostmicrobiome interactions.

- Under specific conditions, EE can parallel the evolution of natural systems.

- EE offers exciting perspectives to discover the function of new genes and probe evolution within communities.

\section{Glossary}

Bow-tie network: architecture of a signalling network where one (or a few) core central regulator is controlled by multiple proteins (input signal) and controls multiple targets (output).

Collateral susceptibility: resistance to one antibiotic increases sensitivity to another.

Cross-resistance: resistance to one antibiotic increases resistance to another.

Experimental evolution: propagation of living organisms in a controlled environment - the laboratory or the field - for several generations (typically, from tens to thousands) allowing to witness the action of natural selection and to investigate evolutionary processes Functional promiscuity: the ability of a protein to perform a secondary activity, often mediated by the inability to distinguish between target molecular substrates (metabolite, DNA, RNA, protein) that have a similar structure.

Fruiting body: multicellular, aggregative structures formed by myxobacteria during nutrient starvation and that contain spores resistant to heat, desiccation or freezing.

Mutators: bacteria with unusually high mutation rates, often as a result of loss of DNA repair genes or expression of error-prone DNA polymerases.

Noise in gene expression: stochastic variations in gene expression level over time and between isogenic cells growing in a homogeneous environment.

Pervasive transcription: describes the fact that large portions of genomes are transcribed, including intergenic regions. 
SRNA: small non-coding RNA with regulatory function

Sub-MIC concentrations: conditions where bacterial growth is not completely stopped by antibiotics.

\section{Outstanding questions:}

- Can we exploit mutations found in EE to characterise genes of unknown function?

- Can EE enlighten mechanisms slowing down the evolution of resistances?

- Can the EE of microbial communities bring new insights into their functional characteristics?

- Can we use within-host EE to identify host factors (and other environmental factors) shaping bacterial evolutionary trajectories during infection?

- Can EE reproduce the emergence of major intracellular symbiotic associations (such as mitochondria in eukaryotic cells, plastids in the green lineage, association between fungi and land plants, obligate endosymbionts of insects)?

\section{Experimental evolution: beyond evolutionary biology}

Artificial breeding of plants and animals played a key role in the maturation of Darwin's theory of evolution by natural selection, but also in the rise of modern human societies (from agriculture to recreational breeding of plants and pets). Non-professional evolutionary biologists performing (sometimes unwittingly) evolution experiments have successfully improved the yield of many biological processes. In contrast, although some evolutionary biologists contemporary of Darwin turned to experimental evolution (EE; see Glossary), the influence of this approach in the development of evolutionary biology only came to prominence in the last few decades [1]. Applied to microbes with short generation times in various conditions (Box 1), EE generated unique biological material (Fig. 1) that was used to probe the evolutionary dynamics of microbial phenotypes $[2,3]$, to decipher the genetic bases of adaptation [4] and to optimize microbial traits for industrial use [5].

In spite of these successes, many biologists remain unaware or unconvinced of the relevance of EE approaches to their research programs. Typical criticisms of EE include the artificial nature of the experimental setups or their simplicity in terms of biotic and abiotic interactions 
[6]. However, this is also true for many excellent lines of research in molecular biology, which depend on the construction of simplified and well-controlled setups. In this article, we argue that experimental evolution has matured into a rich field with a varied set of tools that can help microbiologists to unravel molecular processes underlying adaptive phenotypes. This is independent of the relevance of experimental evolution to reproduce natural adaptive processes, since laboratory phenotypes can be interesting on their own. For example, improving growth rate, yield and recombinant protein production in the laboratory has obvious biotechnological interest [7]. Contemporary microbes can also be used to experiment on the ecological conditions and evolutionary patterns that might have accompanied the evolution of multicellularity millions of years ago [8]. Here, instead of aiming at providing an exhaustive overview of the field (for which we refer the interested reader to recent reviews $[2-4,7-12])$, we use a few selected examples on gene regulation, antibiotic resistance and host-microbiome interactions to illustrate how EE contributes to understand fundamental aspects of molecular biology and helps manipulating natural ecosystems. As the natural inclination of most biologists is to understand if the phenotypes observed in laboratoryevolved mutants are relevant to understand natural processes, we present examples of discrepancies (Box 2), but also increasing evidence of meaningful parallels (Box 3), between EE and natural evolutionary processes. While this review focuses on bacteria, concepts discussed here are also relevant to other organisms, especially viruses or unicellular eukaryotes.

\section{Gene regulation}

A major challenge for modern biology has been to map and understand the logics of gene regulatory networks (GRNs). Outstanding enigmas in molecular biology tackled by EE include how the interactions of transcription factors (TF) with DNA arise and evolve, why they are so hard to identify correctly, and how they form complex networks [13].

The co-option or modulation of bacterial regulatory responses provide a multitude of opportunities for adaptation to new environmental challenges. Indeed, most adaptive mutations in EE are found in regulatory regions or in regulatory genes [4]. Detailed molecular analyses of the associated transcriptional rewiring can reveal new components from signalling 
121 networks, new connections between known regulatory components and new cross-talk 122 between signalling and metabolic pathways. These trends emerged from some of the very 123 early studies in experimental evolution [14, 15]. A prominent example includes the evolution 124 of a new lactose fermentation system in Escherichia coli, via mutations in the egbA $\beta$ 125 galactosidase and its regulator [16]. More recently, EE has uncovered a novel sRNA regulating 126 Myxococcus xanthus fruiting body development. M. xanthus responds to nutrient starvation 127 by generating fruiting bodies containing stress-resistant spores. This behaviour was lost during 128 EE in nutrient-rich liquid medium, but was subsequently re-evolved in alternating cycles of 129 starvation and non-starvation [17]. The point mutation responsible for the re-evolution of 130 fruiting-body formation was found in a previously un-annotated intergenic region, leading to 131 the discovery of the novel sRNA controlling this developmental pathway [18]. Another study 132 established a link between pyrimidine metabolism and the Gac/Rsm signalling pathway, a 133 major determinant of lifestyle switch in Pseudomonas spp. [19] that controls the production 134 of extracellular capsules in Pseudomonas fluorescens. Evolution of $P$. fluorescens under 135 fluctuating environments gave rise to a strain that produces a sub-population of capsulated 136 cells [20]. This phenotype is underpinned by a mutation that decreases pyrimidine 137 biosynthesis [21] and increases ribosome production [22]. That both increased ribosome 138 levels and functional Gac/Rsm signalling are required for heterogeneous capsule production 139 shows that central metabolism (pyrimidine biosynthesis) can alter the output of a two140 component signalling system [22].

142 The plasticity of regulatory regions and transcription factors observed in EE can be recruited 143 to understand fundamental properties of transcriptional control. Low affinity TF-DNA 144 interactions appear to play a critical role in the evolution of GRNs. Yona et al. [23] replaced 145 the lac promoter with random DNA sequences of the same size in E. coli and observed that 146 bacterial growth in the presence of lactose rapidly led to the evolution of functional 147 promoters. A single mutation in synthetic promoters was sufficient to induce substantial 148 expression in most cases (Fig. 2A). The rapid de novo evolution of promoters and the 149 functional promiscuity of transcription factors may explain the intriguing pervasive 150 transcription observed in bacterial genomes [24]. It may also explain the evolutionary 151 plasticity of gene expression in bacteria, since an initially weak binding of a TF on a regulatory 152 region can be reinforced by mutations in the DNA-binding domain. This was observed during 
153 the re-evolution of motility in $P$. fluorescens following the deletion of the master regulator of 154 flagellar synthesis fleQ [25]. Under selection for motility, flagellin expression was restored 155 within $96 \mathrm{~h}$ in a two-step process involving (i) the increased phosphorylation (and thus, 156 activation level) of a nitrogen-related transcriptional regulator with weak (promiscuous) 157 activity on flagellar genes and (ii) a switch-of-function mutation that re-directed 158 transcriptional activity of this regulator from nitrogen uptake to flagellar genes. The plasticity 159 of regulatory interactions can also be associated with noise in gene expression, which has 160 attracted interest in the recent years because it can contribute to phenotypic variability in 161 clonal populations [26]. Wolf et al. [27] showed that libraries of random promoters evolved to 162 produce GFP at high or intermediate expression levels had lower average noise than natural 163 E. coli promoters. This suggests that noise is a selected trait in nature. When the average 164 production of a protein is not optimal in a given environment, a broad (i.e. noisy) distribution 165 of gene expression levels increases the likelihood that some cells of the population express an 166 appropriate amount of this protein. Mathematical modelling suggests that noise can act as a 167 primitive form of gene regulation and pave the way towards more precise regulation [27]. 168 Altogether, these studies illustrate that what may appear to be 'non-optimality' in 169 transcriptional regulation - functional promiscuity and noise - actually drives the evolution of 170 GRNs.

172 EE enables to directly probe systems-level properties of GRNs in an evolutionary context. 173 Witnessing how GRNs are gradually built by evolutionary tinkering (rather than by optimized design) is key to understand their complex architecture and emergent properties, such as robustness, resilience or evolvability [28]. In many cases, transcriptional changes induced by

176 genetic or environmental perturbations are reverted during the early steps of adaptation by adaptive mutations in global regulators that restore cellular homeostasis [29-32]. These results support the idea that transcriptional stability is essential for optimal fitness and can be restored by minimal genetic modifications in global regulators, thus highlighting the resilience of GRNs. However, the topology of GRNs architecture can also benefit bacterial evolvability.

181 Studying the evolution of the bow-tie network controlling flagellar production in $\mathrm{E}$. coli, Ni et 182 al. [33] found that motility in a porous environment (the trait under selection in their 183 experiment) can increase via mutations in multiple target genes. Yet, all adaptive mutations 184 commonly modified the activity of a core sigma-factor checkpoint controlling flagellar gene 
expression. By allowing multiple mutations in independent genes to target the same central regulator of motility, the bow-tie architecture provides evolutionary flexibility for the finetuning of bacterial behaviours. These examples show that EE is a powerful tool to analyse evolutionary properties of GRNs.

\section{Antibiotic resistance}

The emergence of antibiotic resistant bacteria is a remarkable example of human-induced evolutionary process. Resistant bacteria emerge systematically within a few years of the introduction of every novel antibiotic. The consequences are dramatic: 700,000 deaths per year, estimated to increase to over 10 million in the incoming decades (https://amrreview.org/Publications). EE is particularly well-suited to decipher the mechanisms of acquisition of antibiotic resistance given the timescale and intensity of selection in this process. This has produced unexpected novel lines of research. For example, very early studies on the EE of antibiotic resistance kick-started the study of microbial mutagenesis by identifying the first E. coli mutators [34]. The simplicity of tracking resistant bacteria, the societal relevance of the topic, and its implications to molecular genetics and physiology have stimulated research on the mechanisms of acquisition of resistance, on multiple resistance, and on the compensation of the fitness costs of resistance.

Most resistances studied from clinical isolates are strong, because of the obvious medical interest of such cases. EE provides complementary information about resistance in controlled setups where pathways to resistance can be tracked, selection forces tuned, and physiological states controlled for. Notably, EE revealed the important role of low (below the minimum inhibitory concentration; MIC) antibiotic concentrations on the evolution of resistance. These concentrations can be encountered in many types of environments and do not result in cell death, but provide sufficient pressure for the selection of resistant variants and even the evolution of novel mechanisms of resistance [35]. They also accelerate the acquisition of resistance by selecting for mutators [36], and increasing the rates of horizontal gene transfer

215 increase in concentration of rifampicin. By assessing fitness of genetically engineered 216 combinations of mutations from isolates evolved under low rates of environmental change, 
they could show that certain genotypes were evolutionarily inaccessible to evolution under rapid environmental changes. Wistrand-Yuen et al. [39] then showed that Salmonella enterica exposed to sub-MIC levels of streptomycin evolved high-level resistance via mechanisms different from those observed under above-MIC conditions. Sub-MIC resistance evolved through small-effect mutations that combined to confer high-level resistance.

Antibiotics stimulate a set of core responses in bacterial physiology [40], which contributes to explain why resistance to one antibiotic can change the cell's susceptibility to others [41]. Several recent studies detailed the network of cross-resistance and collateral susceptibility resulting from EE of resistance to each of a large range of antibiotics [42-44]. Evolution involving cross-resistance and collateral sensitivity is frequently convergent in $E$. coli, meaning that one can predict to a reasonable extent the antibiotic resistance phenotypes from the genome sequences of the laboratory-evolved lines [45]. Treatments based on alternating drugs with compatible collateral sensitivity profiles could thus be more efficient and lead to slower development of resistances (Fig. 2B; [44]). Several of these studies observed that populations adapted to resist to aminoglycosides show systematically lower fitness in the presence of other types of antibiotics. In molecular terms, this could be the consequence of selection for alterations in the inner membrane potential reducing the uptake of aminoglycoside-related antibiotics, which would simultaneously lower the activity of efflux pumps using proton-motive force [42]. Interestingly, the comparison of interaction networks evolving in conditions of weak and high antibiotic concentration revealed that the strength of selection shapes the acquisition of resistance: cross-resistance tends to be stronger under higher antibiotic concentrations [46]. The stochasticity of evolutionary trajectories leading to resistance to one antibiotic can influence the occurrence of collateral sensitivity [47], calling for a careful assessment of the robustness of this phenomenon when wanting to exploit it in a clinical setting. Together with the studies on the effect of sub-MIC conditions, this suggests that the path towards resistance, involving exposure to high or low antibiotic concentrations, will shape the network of collateral effects of antibiotics.

The many evolution experiments detailing the mutational landscape of antibiotic resistance have systematically revealed rapid partial compensatory evolution of the initial fitness costs of resistance [48-50]. Compensation facilitates the spread and fixation of antibiotic resistant 
lineages. For example, rifampicin resistance in Mycobacterium tuberculosis is costly in 250 laboratory-derived mutants, but multidrug-resistant clinical strains often show no fitness 251 defects [51], because of compensatory mutations [52]. The study of mutants arising in E. coli 252 in the presence of fluoroquinolones showed that compensation of fitness cost associated to 253 antibiotic resistance can lead to bacteria that are as fit as the wild-type susceptible bacteria in 254 the absence of antibiotics [53]. Prolonged colonization of chickens with fluoroquinolone255 resistant Campylobacter jejuni, a somewhat less controlled evolution experiment, revealed 256 that resistant bacteria were also fitter pathogens [54]. Genes that simultaneously increase 257 virulence and resistance may actually be quite common. They were observed in several other 258 pathogens, including Pseudomonas aeruginosa, Acinetobacter baumannii and Vibrio cholerae 259 [55]. Even costly resistance mutations can coexist with other genotypes for hundreds of 260 generations when their high adaptive potential counteracts their initial cost [56]. This means 261 that a multi-step process of slow accumulation of mutations conferring resistance and cost compensation, as expected under sub-MIC conditions, can result in bacteria that are both resistant and fit. This process is strongly dependent on the genetic context of the mutations (epistasis) [57-60]. For example, a comparison of streptomycin and rifampicin double-resistant E. coli with single-resistant clones obtained though EE showed that low-fitness doubleresistant bacteria compensate their cost faster than single-resistant strains thanks to the acquisition of compensatory mutations with larger effects [61]. Surprisingly, some mutations only compensate for double resistance, being neutral or deleterious in single-resistant backgrounds. This means that multiple resistances may not rapidly go away with pauses in the use of the corresponding antibiotics.

\section{Host-microbe interactions}

273 Plants and animals are persistently inhabited by microbes, whose contribution in host health, 274 nutrition and development is increasingly recognized [62, 63]. Elucidating the functional 275 mechanisms and evolutionary potential of host-microbiome interactions is crucial to 276 manipulate this ecosystem and improve host health.

278 Most bacterial pathogens have the remarkable ability to alternate between external environments and specialized host niches. Rapid and coordinated shifts in metabolism, 280 physiology, and virulence factor production in response to environmental changes are 
orchestrated by multilayered and highly complex circuitries that are very difficult to decipher

282 [64]. Adaptation to new hosts, which is generally very rapid in laboratory conditions [65], has 283 the potential to reveal new components of virulence pathways. This was recently highlighted 284 in an evolution experiment aiming at adapting the plant pathogen Ralstonia solanacearum to different host plants [66]. Beneficial mutations improving in planta colonization mainly occurred in a gene, which was named efpR (for enhanced fitness in plants). The efpR gene was shown to encode a transcriptional regulator acting as both a central player of the $R$. solanacearum virulence network and a global catabolic repressor down-regulating the expression of multiple metabolic pathways [67]. Experimental adaptation of $R$. solanacearum to a non-host legume further identified other components of the efpR pathway [68]. Although the genetic bases of virulence in $R$. solanacearum had been amply dissected [69], this pathway had not been previously identified, illustrating how EE coupled with genome resequencing allows identifying novel molecular players of biological functions.

Many ecological transitions towards pathogenic or mutualistic symbiosis include an initial acquisition by horizontal gene transfer (HGT) of mobile genetic elements (MGE) that can provide complex novel traits in a single event of transfer [70,71]. Profound changes in lifestyle may require the remodeling of the metabolic and signaling networks in the recipient genome, a process that may take hundreds to millions of years in natura [72]. The study of this process by the analysis of extant microorganisms can be complemented by EE to pinpoint specific molecular mechanisms that ensure the full expression of acquired traits. This is illustrated by the study of nitrogen-fixing legume symbionts (rhizobia), which evolved through the acquisition of a set of essential symbiotic genes [73]. An experiment was designed to evolve a plant pathogenic bacterium ( $R$. solanacearum) into a legume symbiont under plant (Mimosa) selection pressure. (Fig. 2C). This experiment showed that control mechanisms developed by the host progressively shape bacteria via the multistep selection of compatible bacterial traits [74]. The gradual activation and improvement of the first symbiotic properties, i.e. the induction of root nodules where bacteria fix nitrogen and the infection of these nodules, occurred via the inactivation of the $R$. solanacearum pathogenic type III secretion system and via regulatory rewiring [75-77], demonstrating the requirement of post-HGT modifications to

311 achieve symbiosis in the case of horizontal transfer between distantly related bacteria. Upon 312 experimentally replaying rhizobia evolution, a genetic mechanism was discovered to 
313 accelerate symbiotic evolution [78]. Evolved $R$. solanacearum underwent a transient

314 hypermutagenesis stage that occurred at every inoculation cycle before the cells entered the 315 plant. Investigating the role of imUABC error-prone DNA polymerases present on the 316 transferred symbiotic plasmid provided evidence that this mutagenesis cassette is expressed 317 in stress conditions (outside the host plant), thus increasing genetic diversity and offering 318 more phenotypic diversity to plant selection. imuABC cassettes were found on c.a. $50 \%$ of the 319 symbiotic plasmids supporting the hypothesis that this hypermutagenesis mechanism has 320 facilitated the evolution of new rhizobia in natura. After 400 generations, mutualistic nitrogen 321 fixation was not achieved, possibly because time was too short. Yet another evolution 322 experiment performed with the natural symbiont of Mimosa (Cupriavidus taiwanensis) 323 showed that, thanks to host sanctions occurring at the post-infection level, rare nitrogen-fixing 324 symbionts (that may arise via mutation during evolution) progressively invade a population 325 dominated by non-fixing bacteria, with a probability that depends on ecological factors [79]. 326 This provided a better understanding of the spread of the mutualistic trait during natural 327 evolution [73].

Plant and animal microbiomes are composed of complex and dynamic bacterial consortia 330 whose interspecific interactions have implications for the host [80]. The importance of 331 bacterial antagonism for the evolution of infection was demonstrated in a tri-partite 332 interaction between Caenorhabditis elegans and two bacterial pathogens. In a first EE, King et 333 al. [81] showed that mildly pathogenic bacteria (Enterococcus faecalis) living in worms rapidly 334 evolved increased competitiveness against a more virulent pathogen (Staphylococcus aureus). 335 This reduced the mortality caused by S. aureus infections. The mechanistic basis for protection 336 was an increased production by $E$. faecalis of antimicrobial reactive oxygen species directly 337 affecting pathogen growth. Although this broad-spectrum defense mechanism was not novel, 338 it showed that microbes living within a host can become mutualists in response to infection 339 by other pathogens. A subsequent EE showed that microbe-microbe interactions within hosts 340 can drive the evolution of pathogens. To limit E. faecalis colonization, which exploits the costly 341 siderophores of $S$. aureus, the latter evolved to produce less siderophores [82]. Since 342 siderophore production contributes to virulence by improving pathogen growth in iron343 limited hosts, its diminution leads to less virulent clones. Hence, bacterial antagonistic 
344 interactions can modulate the production of virulence factors, and consequently influence 345 how the microbiome impacts the host.

\section{Concluding remarks and future perspectives}

348 EE has already provided a wealth of information on the molecular events (see [83] for 349 additional examples) and the conditions driving phenotypic adaptation in a number of model 350 biological systems. We expect that many more discoveries will follow. For practical purposes, 351 characterisation of adaptive mutations has usually focused on known genes and pathways. A 352 large number of adaptive mutations found in genes of unknown function remains to be 353 analysed, representing a challenging untapped reservoir of new discoveries. The development 354 of cross-experiments databases (such as the recent ALEdb [84]) may help identifying 355 promising candidate genes to initiate such studies. Moreover, the introduction of novel non356 model bacteria allows EE to tackle different biological questions, e.g. studying the evolution 357 and functioning of microbial communities (see Outstanding Questions). EE can also provide 358 an alternative to genetic screens for organisms that are not genetically amenable.

359 EE can be used to study mechanisms of evolution, and their underlying molecular biology, 360 independently of the events that actually took place in the natural history of the species. Yet, 361 it would be of remarkable interest to use EE as a tool to test hypothesis about evolution in 362 nature, especially in cases where the study of natural populations only provides limited 363 insights into ancient processes. EE is not necessarily relevant in this context because of its 364 simplified setups, and numerous reports revealed differences between EE and natural 365 processes (Box 2). Nevertheless, there is a growing number of reported parallels between 366 natural and EE, especially in studies where the latter tried to match more closely the 367 conditions of natural evolution (Box 3). The extent to which EE studies can mirror the natural 368 evolution of bacterial traits is likely to be a fertile area for future research.

369 On the technological side, recent genome editing technologies, including CRISPR-Cas or 'deep 370 mutational scanning', tremendously accelerate the exploration of genotype-phenotype 371 landscapes [10]. Moreover, new cultivation procedures (particularly those based on 372 micro/millifluidics $[85,86]$ ) will allow large scale, automated evolution experiments, and their 373 genetic analysis can be facilitated by DNA barcoding [10]. With continuously decreasing 374 sequencing cost, the EE field is therefore ripe for appropriation by molecular microbiologists 375 coming with imaginative selective regimes, original microbial strains or communities, and 
novel biological questions. More generally, the adoption of EE by a wider community of microbiologists could accelerate the march towards the much-needed synthesis of molecular and evolutionary approaches [87-89].

\section{Box 1: The many faces of experimental evolution}

Experimental evolution can be performed under a wide range of experimental settings adapted to the biological question of interest. Details on the design of these studies were reviewed elsewhere $[3,90]$. As an attempt to classify the most common EE practices, one can distinguish levels of complexity along two criteria: environmental conditions and starting biological material (Fig. 1).

Starting biological material: Wild-type bacterial strains are commonly used to start evolution experiments. Although in theory any cultivable strain can be used, most works focus on fastgrowing genetically tractable model bacteria. Other studies employ modify-and-evolve approaches where genetic engineering is used to delete [32], introduce a trait $[23,27,75]$ or modify a gene or the whole genome $[91,92]$, and is followed by EE to understand how the system evolves. These approaches profit enormously from the recent developments in synthetic biology and CRISPR-based technologies. Comparative evolution studies are typically performed by evolving several independent lines from the same ancestor in parallel, but can also involve different strains/species exposed to the same conditions [93]. Complexity in starting material can be increased by putting together different bacterial species [94, 95], or complex communities [96]. In these cases, experimentalists can follow either the evolution of one of the organisms or the co-evolution of multiple organisms.

Environmental complexity: Following the trends set by the long-term evolution experiment (LTEE), most microbial evolution experiments are performed in very simple growth conditions, e.g. in shaken Erlenmeyer flasks [97] or in chemostats [98], with a variety of volumes, time delay and volume of transfer between subsequent cycles (flasks) or dilution rates (chemostats). A number of studies used more complex environments. Spatially structured environments are generated in static liquid cultures (creating an oxygen gradient [99]), on solid supports within liquid medium [100] or on solid surfaces (agar plates [25]). In this case, micro-organisms deplete resources locally, leading to differences between patches. Growth on solid media or in liquid meta-populations also helps manipulating genetic assortment 
408 between neighbouring cells, a condition often used to explore the evolution of social 409 behaviours [101-103]. The strength of selective pressures can be adjusted during the 410 experiment by varying antibiotic concentrations in a continuous culture [58], creating 411 chemical gradients in agar plates [104], or by varying conditions between successive growth 412 cycles [20,43]. Finally, some studies mimic natural conditions more closely by employing more 413 complex settings, such as eukaryotic hosts, to study the evolution of mutualists or pathogens $414 \quad[66,77,81,105-107]$.

\section{Box 2: Divergences between EE and natural evolution.}

417 Many EE studies identified patterns of molecular evolution with large excesses of non418 synonymous adaptive mutations, whereas natural populations systematically show a 419 predominance of synonymous substitutions caused by purifying selection on protein 420 sequences [108]. This may result from the joint effects of the simple continuous unidirectional 421 selective pressures applied in many EE together with the use of conditions free from most 422 other constraints that bacteria endure in natural environments. The same reasons may explain 423 why core genes tend to accumulate few substitutions in natural populations, but evolve faster 424 in the LTEE [109]. The contrast between evolutionary patterns is particularly striking for the 425 RNA polymerase gene $r p o B$ that often accumulates adaptive mutations in in vitro EE 426 experiments but is extremely conserved in natural evolution [4]. Interestingly, a recent $E$. coli 427 EE study in the mouse gut, a more natural environment, showed lower rates of evolution and 428 no mutations in $r p o B$ [110]. Similar discrepancies were found by a study where $P$. aeruginosa 429 adaptation to the airways of cystic fibrosis patients during over 200,000 generations resulted 430 in limited genetic diversification. In contrast with in vitro $\mathrm{EE}$, the in vivo process revealed an 431 initial period of adaptive mutations followed by a period with the more usual pattern of 432 dominance of purifying selection [111]. Another reason for the excess of non-synonymous 433 adaptive mutations in EE is the lack of sexual exchanges with distant strains or species in most 434 EE setups. This prevents the income of adaptive changes by horizontal gene transfer and 435 results in adaptive mutations touching key processes that are highly conserved in nature. For 436 example, many traits are lost in EE because they are costly in simplified setups, but they are 437 under selection - and thus conserved - in nature [112]. A striking example of this contrast is 438 given by the frequent evolution of mutators during phage-bacteria co-evolution in simple 
experiments, which was not observed in complex environments closer to natural conditions $440[113,114]$.

\section{Box 3: Parallels between EE and natural evolution.}

443 The use of more complex setups in EE has found interesting parallels with analogous processes 444 in natural history. For example, diversification of $M$. xanthus during EE led to genetic diversity close to those identified in natural populations sampled from small parcels in the soil [115].

446 EE of Burkholderia cenocepacia in biofilms revealed a wealth of mutations associated with its adaptation and diversification of which four broad classes were also found in clinical isolates of Burkholderia dolosa and P. aeruginosa cystic fibrosis patients, suggesting a parallelism between adaptation to the biofilm lifestyle and lung colonization [100]. A recent study aimed at comparing directly the patterns of evolution of resistance to colistin in $P$. aeruginosa using both laboratory EE and the analysis of four clinical isolates from a single cystic fibrosis patient

452 (sampled within a period of three months) [60]. This revealed a complex, multistep adaptation 453 process requiring epistatic mutations in several loci where parallels between the natural and experimental processes could be identified: all resistant mutants were mutators and the evolution of resistance occurred through mutations in prmB, part of the PrmAB twocomponent system. This shows that processes at comparable time scales requiring a relatively straightforward adaptation process can reveal significant parallels. Another study combining detailed phenotypic characterization and mathematical modelling showed that high mutational supply, influenced by population and bottleneck sizes, was a key parameter

460 favoring parallelism between laboratory and natural evolution of ciprofloxacin resistance in $E$. 461 coli [116].

462 More complex adaptation processes were investigated by evolving three clones of 463 Lactococcus lactis from a plant isolate to the dairy niche [117]. Gene expression differences 464 between the parental and the dairy strain were maximal at an operon encoding an $A B C$ 465 transporter that was 350 times more expressed in the dairy strain. Interestingly this operon 466 was also expressed at higher level in two of the three adapted strains. An even more radical $467 \mathrm{EE}$, leading to a change in lifestyle from a plant pathogen to a rhizobial mutualist upon 468 acquisition of a large plasmid carrying the symbiosis genes and after a few hundreds of 469 generations (see Main Text), also showed striking parallels to the natural process that took 
470

471

472

473

474

475

476

477

478

479

480

481

482

483

484

485

486

487

488

489

490

491

492

493

494

495

496 having acquired a single mutation (black star) exhibit on average $50 \%$ of the wild-type (WT) 497 activity.

place over more than 10 million years [118]. In spite of the radically different time-span of the evolutionary processes and degree of achievement of the symbioses, adaptation was accompanied in both cases by an overall pattern of purifying selection. Both natural and experimental processes showed very little signal of adaptation in the fast-evolving symbiotic plasmid, whereas many adaptive mutations took place in the genetic background of the bacteria, including mutations that led to the co-option of the same quorum-sensing system in both processes. These works show that EE reveals significant parallels to natural history when it mimics key conditions of the natural processes.

\section{Figure legends:}

Fig. 1: Experimental evolution: a source of biological material available for phenotypic and genotypic analysis.

Traditionally, only extant or very recent populations resulting from millions of years of natural evolution are available for analysis. EE allows the analysis of all steps of adaptation during years of accelerated evolution in controlled conditions, thanks to frozen fossil records. Recently, the intensive genomic sampling of variants in natural populations provides data that can be compared with that of EE. Points represent available naturally- or experimentallyevolved bacterial clones/populations.

Fig. 2. Selected evolution experiments having contributed to advances in gene regulation (A), antibiotic resistance (B) and host-microbe interactions (C).

93 A. Evolution of promoters [23]. Left: The lac promoter was replaced by random sequences in

94 E. coli. Bacteria were evolved by serial dilutions in $0.05 \%$ glycerol (utilized by the strain) and

$950.2 \%$ lactose (originally not utilized). Right: Following laboratory selection, $~ 60 \%$ of promoters 
B. Evolution of collateral sensitivity and its use to design new therapeutic strategies [44]. Left: Bacteria selected to resist a given antibiotic $(X)$ reproducibly display an increased (crossresistance), unchanged or decreased (collateral sensitivity) resistance to other antibiotics (e.g., A) compared to their WT ancestor. Right: Patterns of collateral sensitivity can be exploited by cycling antibiotic treatments that accelerate eradication of bacterial pathogens. CFU: colony forming units.

C. Evolution of new legume symbionts [77, 78]. The symbiosis plasmid of Cupriavidus taiwanensis was introduced into Ralstonia solanacearum, generating a non-nodulating protorhizobium ( $\mathrm{Nod}^{-}$) that was further evolved using serial cycles of co-culture with Mimosa pudica, the natural host of $C$. taiwanensis. The symbiosis plasmid possesses the essential nod and nif/fix genes required for nodulation and nitrogen fixation. In addition, it contains imuABC genes encoding stress-responsive error-prone DNA polymerases that transiently elevated the mutation rate of bacteria growing in the rhizosphere. In only 16 cycles (c.a. 400 generations) the ancestral proto-rhizobium, which was only able to induce root hair curling $\left(\mathrm{Hac}^{+}\right)$allowing the formation of infection sites, successively acquired the capacity to enter the root and form nodules $\left(\mathrm{Nod}^{+}\right)$, extracellularly infect nodules $\left(E-\operatorname{lnf}^{+}\right)$, intracellularly invade nodules $\left(1-\operatorname{lnf} f^{+}\right)$and massively invade nodule cells $\left(I-\operatorname{lnf}^{++}\right)$, via genome remodeling. Stars symbolize mutations. Bacteria are represented in blue in nodules. Adapted from references [74, 78].

\section{Fig. 1 for Box 1: Experimental settings in EE}

518 Examples of experiments using biological material or environments exhibiting increasing 519 levels of complexity.

\section{Acknowledgements}

522 We apologize to colleagues whose work could not be cited here due to space limitations. PR 523 has received the support of the EU in the framework of the Marie-Curie FP7 COFUND People 524 Programme, through the award of an AgreenSkills+ fellowship under grant agreement $\mathrm{n}^{\circ} \mathrm{FP7}$ 525 609398. CMB, EPCR, and PR were supported by funds from the French National Research 526 Agency (ANR-16-CE20-0011-01). CMB and PR were supported by the French Laboratory of 
Excellence project 'TULIP' (ANR-10-LABX-41). EPCR thanks lab members for discussions on this

\section{References:}

1. Adams, J. and Rosenzweig, F. (2014) Experimental microbial evolution: History and conceptual underpinnings. Genomics 104 (6), 393-398.

2. Lenski, R.E. (2017) Experimental evolution and the dynamics of adaptation and genome evolution in microbial populations. ISME J. 11 (10), 2181-2194.

3. Van den Bergh, B. et al. (2018) Experimental design, population dynamics, and diversity in microbial experimental evolution. Microbiol. Mol. Biol. Rev. 82 (3), e00008-18.

4. Long, A. et al. (2015) Elucidating the molecular architecture of adaptation via evolve and resequence experiments. Nat. Rev. Genet. 16 (10), 567-582.

540 5. Chen, K.Q. and Arnold, F.H. (1993) Tuning the activity of an enzyme for unusual environments: Sequential random mutagenesis of subtilisin E for catalysis in dimethylformamide. Proc. Natl. Acad. Sci. U.S.A. 90 (12), 5618-5622.

7. Dragosits, M. and Mattanovich, D. (2013) Adaptive laboratory evolution - principles and applications for biotechnology. Microb. Cell Fact. 12, 64.

8. Rainey, P.B. et al. (2017) Darwin was right: Where now for experimental evolution? Curr. Opin. Genet. Dev. 47, 102-109.

9. Martin, M. et al. (2016) Laboratory evolution of microbial interactions in bacterial biofilms. J. Bacteriol. 198 (19), 2564-2571.

550 10. Bruger, E.L. and Marx, C.J. (2018) A decade of genome sequencing has revolutionized studies of 551 experimental evolution. Curr. Opin. Microbiol. 45, 149-155.

552 11. Hoang, K.L. et al. (2016) Experimental evolution as an underutilized tool for studying beneficial 553 animal-microbe interactions. Front. Microbiol. 7, 1444.

554 12. Blount, Z.D. et al. (2018) Contingency and determinism in evolution: Replaying life's tape. Science $555362(6415)$, eaam5979.

556 13. Inukai, S. et al. (2017) Transcription factor-DNA binding: Beyond binding site motifs. Curr. Opin. Genet. Dev. 43, 110-119.

14. Mortlock, R.P. (1982) Metabolic acquisitions through laboratory selection. Annu. Rev. Microbiol. 36, 259-284.

15. Clarke, P.H. and Drew, R. (1988) An experiment in enzyme evolution. Studies with Pseudomonas aeruginosa amidase. Bioscience Rep. 8 (2), 103-120.

16. Hall, B.G. and Clarke, N.D. (1977) Regulation of newly evolved enzymes. III Evolution of ebg repressor during selection for enhanced lactase activity. Genetics 85 (2), 193-201.

17. Fiegna, F. et al. (2006) Evolution of an obligate social cheater to a superior cooperator. Nature 441 (7091), 310-314.

18. Yu, Y.T.N. et al. (2010) Adaptive evolution of an sRNA that controls Myxococcus development. Science 328 (5981), 993-993.

19. Lapouge, K. et al. (2008) Gac/Rsm signal transduction pathway of gamma-proteobacteria: from RNA recognition to regulation of social behaviour. Mol. Microbiol. 67 (2), 241-253.

20. Beaumont, H.J.E. et al. (2009) Experimental evolution of bet hedging. Nature 462 (7269), 90-93.

21. Gallie, J. et al. (2015) Bistability in a metabolic network underpins the de novo evolution of colony switching in Pseudomonas fluorescens. PLoS Biol. 13 (3), e1002109.

22. Remigi, P. et al. (2018) Ribosome provisioning activates a bistable switch coupled to fast exit from stationary phase. bioRxiv. doi.org/10.1101/244129

$5769,1530$. 
24. Wade, J.T. and Grainger, D.C. (2014) Pervasive transcription: Illuminating the dark matter of bacterial transcriptomes. Nat. Rev. Microbiol. 12 (9), 647-653.

579 25. Taylor, T.B. et al. (2015) Evolutionary resurrection of flagellar motility via rewiring of the nitrogen 580 regulation system. Science 347 (6225), 1014-1017.

581 26. Raser, J.M. and O'Shea, E.K. (2005) Noise in gene expression: Origins, consequences, and control. 582 Science 309 (5743), 2010-2013.

583 27. Wolf, L. et al. (2015) Expression noise facilitates the evolution of gene regulation. elife 4, e05856.

584 28. Sorrells, T.R. and Johnson, A.D. (2015) Making sense of transcription networks. Cell 161 (4), 714585723.

586 29. Stoebel, D.M. et al. (2009) Compensatory evolution of gene regulation in response to stress by Escherichia coli lacking RpoS. PLoS Genet. 5 (10), e1000671.

30. Carroll, S.M. and Marx, C.J. (2013) Evolution after introduction of a novel metabolic pathway consistently leads to restoration of wild-type physiology. PLoS Genet. 9 (4), e1003427.

31. Rodriguez-Verdugo, A. et al. (2016) First-step mutations during adaptation restore the expression of hundreds of genes. Mol. Biol. Evol. 33 (1), 25-39.

32. McCloskey, D. et al. (2018) Evolution of gene knockout strains of E. coli reveal regulatory architectures governed by metabolism. Nat. Commun. 9, 3796.

33. Ni, B. et al. (2017) Evolutionary remodeling of bacterial motility checkpoint control. Cell Rep. 18 (4), 866-877.

34. Treffers, H.P. et al. (1954) A factor (or mutator gene) influencing mutation rates in Escherichia coli. Proc. Natl. Acad. Sci. U.S.A. 40 (11), 1064-1071.

35. Gullberg, E. et al. (2011) Selection of resistant bacteria at very low antibiotic concentrations. PLoS Pathog. 7 (7), e1002158.

36. Gutierrez, A. et al. (2013) $\beta$-lactam antibiotics promote bacterial mutagenesis via an RpoSmediated reduction in replication fidelity. Nat. Commun. 4, 1610.

37. Lopez, E. and Blazquez, J. (2009) Effect of subinhibitory concentrations of antibiotics on intrachromosomal homologous recombination in Escherichia coli. Antimicrob. Agents Chemother. 53 (8), 3411-3415.

38. Lindsey, H.A. et al. (2013) Evolutionary rescue from extinction is contingent on a lower rate of environmental change. Nature 494 (7438), 463-467.

39. Wistrand-Yuen, E. et al. (2018) Evolution of high-level resistance during low-level antibiotic exposure. Nat. Commun. 9, 1599.

40. Mathieu, A. et al. (2016) Discovery and function of a general core hormetic stress response in $E$. coli Induced by sublethal concentrations of antibiotics. Cell Rep. 17 (1), 46-57.

41. Girgis, H.S. et al. (2009) Genetic architecture of intrinsic antibiotic susceptibility. PLoS One 4 (5), e5629.

42. Lazar, V. et al. (2013) Bacterial evolution of antibiotic hypersensitivity. Mol. Syst. Biol. 9, 700.

43. Kim, S. et al. (2014) Alternating antibiotic treatments constrain evolutionary paths to multidrug resistance. Proc. Natl. Acad. Sci. U.S.A. 111 (40), 14494-14499.

44. Imamovic, L. and Sommer, M.O.A. (2013) Use of collateral sensitivity networks to design drug cycling protocols that avoid resistance development. Sci. Transl. Med. 5, 204 ra132.

45. Lazar, V. et al. (2014) Genome-wide analysis captures the determinants of the antibiotic crossresistance interaction network. Nat. Commun. 5, 4352.

46. Oz, T. et al. (2014) Strength of selection pressure is an important parameter contributing to the complexity of antibiotic resistance evolution. Mol. Biol. Evol. 31 (9), 2387-2401.

47. Nichol, D. et al. (2019) Antibiotic collateral sensitivity is contingent on the repeatability of evolution. Nat. Commun. 10 (1), 334.

48. Reynolds, M.G. (2000) Compensatory evolution in rifampin-resistant Escherichia coli. Genetics 156 (4), 1471-1481.

49. Nguyen, T.N.M. et al. (1989) Effects of carriage and expression of the Tn10 tetracycline-resistance operon on the fitness of Escherichia coli K12. Mol. Biol. Evol. 6 (3), 213-225. 
50. Brandis, G. et al. (2012) Fitness-compensatory mutations in rifampicin-resistant RNA polymerase. Mol. Microbiol. 85 (1), 142-151.

630 51. Gagneux, S. et al. (2006) The competitive cost of antibiotic resistance in Mycobacterium 631 tuberculosis. Science 312 (5782), 1944-1946.

632 52. Comas, I. et al. (2012) Whole-genome sequencing of rifampicin-resistant Mycobacterium 633 tuberculosis strains identifies compensatory mutations in RNA polymerase genes. Nat. Genet. 44 (1), $634 \quad 106-110$.

635 53. Marcusson, L.L. et al. (2009) Interplay in the selection of fluoroquinolone resistance and bacterial 636 fitness. PLoS Pathog. 5 (8), e1000541.

637 54. Luo, N.D. et al. (2005) Enhanced in vivo fitness of fluoroquinolone-resistant Campylobacter jejuni in the absence of antibiotic selection pressure. Proc. Natl. Acad. Sci. U.S.A. 102 (3), 541-546. 55. Roux, D. et al. (2015) Fitness cost of antibiotic susceptibility during bacterial infection. Sci. Transl. Med. 7, 297 ra114.

56. de Sousa, J.M. et al. (2015) Potential for adaptation overrides cost of resistance. Future Microbiol. 10 (9), 1415-1431.

57. Weinreich, D.M. et al. (2006) Darwinian evolution can follow only very few mutational paths to fitter proteins. Science 312 (5770), 111-114.

58. Toprak, E. et al. (2011) Evolutionary paths to antibiotic resistance under dynamically sustained drug selection. Nat. Genet. 44, 101-105.

59. Trindade, S. et al. (2009) Positive epistasis drives the acquisition of multidrug resistance. PLoS Genet. 5 (7), e1000578.

60. Jochumsen, N. et al. (2016) The evolution of antimicrobial peptide resistance in Pseudomonas aeruginosa is shaped by strong epistatic interactions. Nat. Commun. 7, 13002.

61. de Sousa, J.M. et al. (2017) Multidrug-resistant bacteria compensate for the epistasis between resistances. PLOS Biol. 15 (4), e2001741.

62. Bai, Y. et al. (2015) Functional overlap of the Arabidopsis leaf and root microbiota. Nature 528, 364369.

63. Huttenhower, C. et al. (2012) Structure, function and diversity of the healthy human microbiome. Nature 486 (7402), 207-214.

64. McAdams, H.H. et al. (2004) The evolution of genetic regulatory systems in bacteria. Nat. Rev. Genet. 5 (3), 169-178.

65. Ebert, D. (1998) Experimental evolution of parasites. Science 282 (5393), 1432-1435.

66. Guidot, A. et al. (2014) Multihost experimental evolution of the pathogen Ralstonia solanacearum unveils genes involved in adaptation to plants. Mol. Biol. Evol. 31 (11), 2913-2928.

67. Perrier, A. et al. (2016) Enhanced in planta fitness through adaptive mutations in EfpR, a dual regulator of virulence and metabolic functions in the plant pathogen Ralstonia solanacearum. PLoS Pathog. 12 (12), e1006044.

68. Capela, D. et al. (2017) Recruitment of a lineage-specific virulence regulatory pathway promotes intracellular infection by a plant pathogen experimentally evolved into a legume symbiont. Mol. Biol. Evol. 34 (10), 2503-2521.

69. Genin, S. and Denny, T.P. (2012) Pathogenomics of the Ralstonia solanacearum species complex. Annu. Rev. Phytopathol. 50, 67-89.

70. Dobrindt, U. et al. (2004) Genomic islands in pathogenic and environmental microorganisms. Nat. Rev. Microbiol. 2 (5), 414-424.

71. Ochman, H. and Moran, N.A. (2001) Genes lost and genes found: Evolution of bacterial pathogenesis and symbiosis. Science 292 (5519), 1096-1098.

72. Lercher, M.J. and Pál, C. (2008) Integration of horizontally transferred genes into regulatory interaction networks takes many million years. Mol. Biol. Evol. 25 (3), 559-67.

73. Remigi, P. et al. (2016) Symbiosis within ymbiosis: Evolving nitrogen-fixing legume symbionts. Trends Microbiol. 24 (1), 63-75.

74. Masson-Boivin, C. and Sachs, J.L. (2018) Symbiotic nitrogen fixation by rhizobia-the roots of a success story. Curr. Opin. Plant Biol. 44, 7-15. 
75. Marchetti, M. et al. (2010) Experimental evolution of a plant pathogen into a legume symbiont. PLoS Biol. 8 (1), e1000280.

682 76. Guan, S.H. et al. (2013) Experimental evolution of nodule intracellular infection in legume 683 symbionts. ISME J. 7 (7), 1367-1377.

684 77. Marchetti, M. et al. (2014) Shaping bacterial symbiosis with legumes by experimental evolution. 685 Mol. Plant Microbe Interact. 27 (9), 956-964.

686 78. Remigi, P. et al. (2014) Transient hypermutagenesis accelerates the evolution of legume endosymbionts following horizontal gene transfer. PLoS Biol. 12 (9), e1001942.

79. Daubech, B. et al. (2017) Spatio-temporal control of mutualism in legumes helps spread symbiotic nitrogen fixation. elife 6, e28683.

80. Garcia-Bayona, L. and Comstock, L.E. (2018) Bacterial antagonism in host-associated microbial communities. Science 361 (6408), eaat2456.

81. King, K.C. et al. (2016) Rapid evolution of microbe-mediated protection against pathogens in a worm host. ISME J. 10 (8), 1915-1924.

82. Ford, S.A. et al. (2016) Microbe-mediated host defence drives the evolution of reduced pathogen virulence. Nat. Commun. 7, 13430.

83. Yi, X. (2017) Experimental evolution and proximate mechanisms in biology. Synth. Syst. Biotechnol. $2(4), 253-258$.

84. Phaneuf, P.V. et al. (2019) ALEdb 1.0: a database of mutations from adaptive laboratory evolution experimentation. Nucleic Acids Res. 47(D1), D1164-D1171.

85. Rotem, A. et al. (2018) Evolution on the biophysical fitness landscape of an RNA virus. Mol. Biol. Evol. 35 (10), 2390-2400.

86. Cottinet, D. et al. (2016) Lineage tracking for probing heritable phenotypes at single-cell resolution. PLoS One 11 (4), e0152395.

87. Upson, J.L. et al. (2018) The coming of age of EvoMPMI: evolutionary molecular plant-microbe interactions across multiple timescales. Curr. Opin. Plant Biol. 44, 108-116.

88. Dean, A.M. and Thornton, J.W. (2007) Mechanistic approaches to the study of evolution: the functional synthesis. Nat. Rev. Genet. 8 (9), 675-688.

89. Alizon, S. and Methot, P.O. (2018) Reconciling Pasteur and Darwin to control infectious diseases. PLoS Biol. 16 (1), e2003815.

90. Cooper, V.S. (2018) Experimental evolution as a high-throughput screen for genetic adaptations. Msphere 3 (3), e00121-18.

91. Wannier, T.M. et al. (2018) Adaptive evolution of genomically recoded Escherichia coli. Proc. Natl. Acad. Sci. U.S.A. 115 (12), 3090-3095.

92. Kacar, B. et al. (2017) Experimental evolution of Escherichia coli harboring an ancient translation protein. J. Mol. Evol. 84 (2-3), 69-84.

93. Gifford, D.R. et al. (2018) Identifying and exploiting genes that potentiate the evolution of antibiotic resistance. Nat. Ecol. Evol. 2 (6), 1033-1039.

94. Hillesland, K.L. and Stahl, D.A. (2010) Rapid evolution of stability and productivity at the origin of a microbial mutualism. Proc. Natl. Acad. Sci. U.S.A. 107 (5), 2124-2129.

95. Hansen, S.K. et al. (2007) Evolution of species interactions in a biofilm community. Nature 445 (7127), 533-536.

96. Barroso-Batista, J. et al. (2015) Adaptive immunity increases the pace and predictability of evolutionary change in commensal gut bacteria. Nat. Commun. 6, 8945.

97. Lenski, R.E. et al. (1991) Long-term experimental evolution in Escherichia coli. 1. Adaptation and divergence during 2,000 generations. Am. Nat. 138 (6), 1315-1341.

98. Notley-McRobb, L. et al. (2002) rpoS mutations and loss of general stress resistance in Escherichia coli populations as a consequence of conflict between competing stress responses. J. Bacteriol. 184 (3), 806-811.

99. Rainey, P.B. and Travisano, M. (1998) Adaptive radiation in a heterogeneous environment. Nature 394 (6688), 69-72. 
100. Traverse, C.C. et al. (2013) Tangled bank of experimentally evolved Burkholderia biofilms reflects selection during chronic infections. Proc. Natl. Acad. Sci. U.S.A. 110 (3), E250-E259. 101. Bachmann, H. et al. (2013) Availability of public goods shapes the evolution of competing metabolic strategies. Proc. Natl. Acad. Sci. U.S.A. 110 (35), 14302-14307.

102. Hammerschmidt, K. et al. (2014) Life cycles, fitness decoupling and the evolution of multicellularity. Nature 515 (7525), 75-79.

103. Chao, L. and Levin, B.R. (1981) Structured habitats and the evolution of anticompetitor toxins in bacteria. Proc. Natl. Acad. Sci. U.S.A. 78 (10), 6324-6328. 104. Baym, M. et al. (2016) Spatiotemporal microbial evolution on antibiotic landscapes. Science 353 (6304), 1147-1151. 105. Jansen, G. et al. (2015) Evolutionary transition from pathogenicity to commensalism: Global regulator mutations mediate fitness gains through virulence attenuation. Mol. Biol. Evol. 32 (11), 28832896.

744 106. Giraud, A. et al. (2001) Costs and benefits of high mutation rates: Adaptive evolution of bacteria in the mouse gut. Science 291 (5513), 2606-2608. 107. Pankey, M.S. et al. (2017) Host-selected mutations converging on a global regulator drive an adaptive leap towards symbiosis in bacteria. elife 6, e24414.

108. Rocha, E.P.C. (2018) Neutral theory, microbial practice: Challenges in bacterial population genetics. Mol. Biol. Evol. 35 (6), 1338-1347.

109. Maddamsetti, R. et al. (2017) Core genes evolve rapidly in the Long-Term Evolution Experiment with Escherichia coli. Genome Biol. Evol. 9 (4), 1072-1083.

110. Lescat, M. et al. (2017) Using long-term experimental evolution to uncover the patterns and determinants of molecular evolution of an Escherichia coli natural isolate in the streptomycin-treated mouse gut. Mol. Ecol. 26 (7), 1802-1817.

111. Yang, L. et al. (2011) Evolutionary dynamics of bacteria in a human host environment. Proc. Natl. Acad. Sci. U.S.A. 108 (18), 7481-7486.

112. Behe, M.J. (2010) Experimental evolution, loss-of-function mutations, and "the first rule of adaptive evolution". Q. Rev. Biol. 85 (4), 419-445.

759 113. Pal, C. et al. (2007) Coevolution with viruses drives the evolution of bacterial mutation rates. $760 \quad$ Nature 450 (7172), 1079-1081.

761 114. Gomez, P. and Buckling, A. (2013) Coevolution with phages does not influence the evolution of bacterial mutation rates in soil. ISME J. 7 (11), 2242-2244.

763

764 115. Rendueles, O. and Velicer, G.J. (2017) Evolution by flight and fight: diverse mechanisms of adaptation by actively motile microbes. ISME J. 11 (2), 555-568.

765 116. Huseby, D.L. et al. (2017) Mutation supply and relative fitness shape the genotypes of ciprofloxacin-resistant Escherichia coli. Mol. Biol. Evol. 34 (5), 1029-1039. 117. Bachmann, H. et al. (2012) Microbial domestication signatures of Lactococcus lactis can be reproduced by experimental evolution. Genome Res. 22 (1), 115-124. 


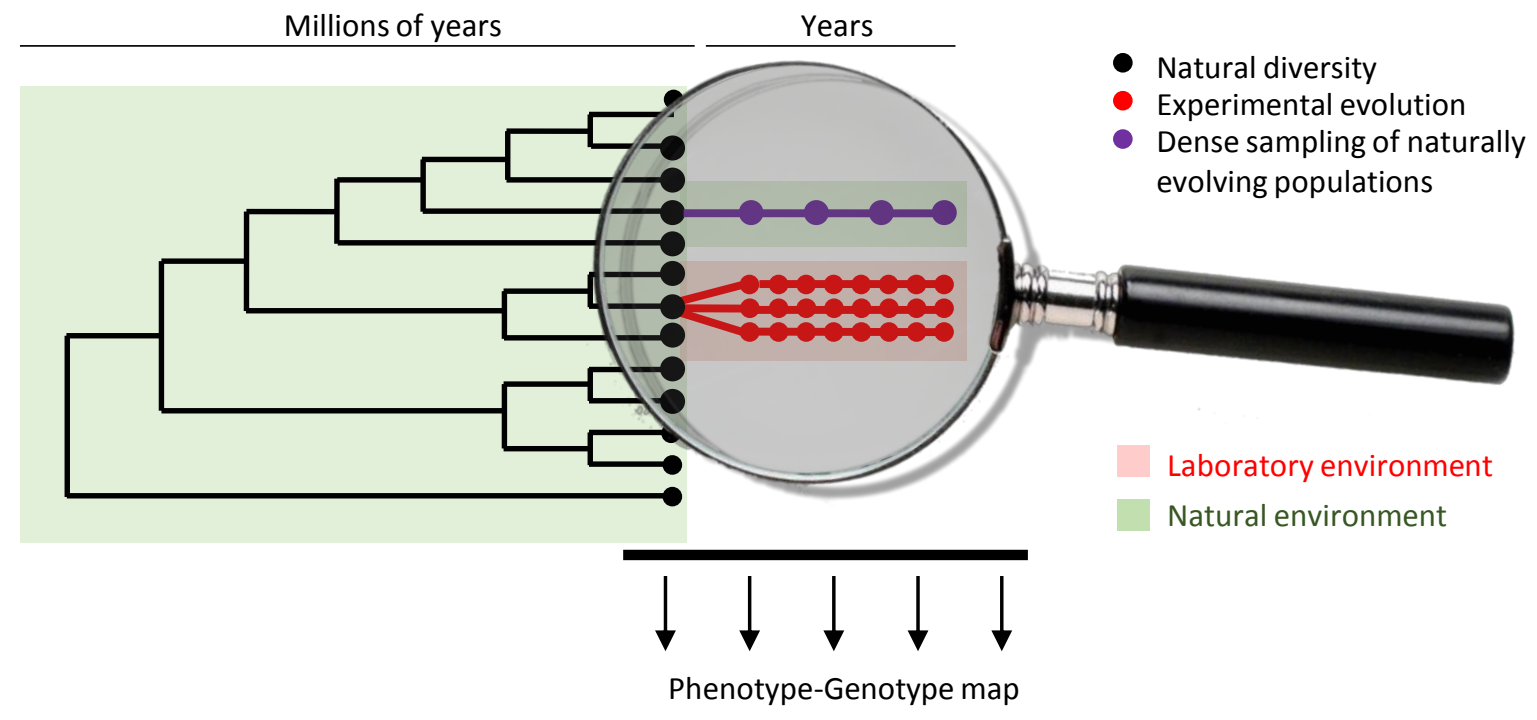

- Natural diversity

Experimental evolution

Dense sampling of naturally evolving populations

Laboratory environment

Natural environment

Phenotype-Genotype map 
A Random promoter library

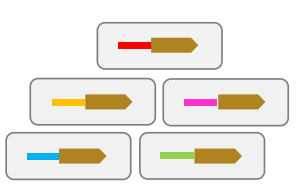

promoter lacz

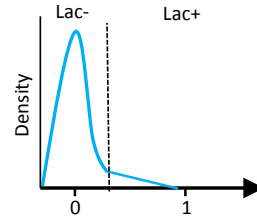

Expression level relative to WT
Evolved promoters
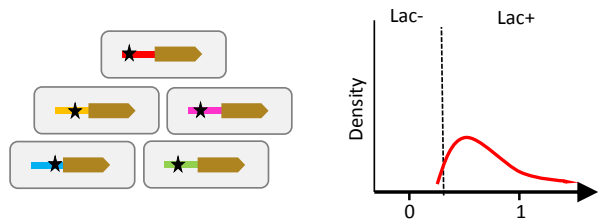

Expression level relative to WT

B

Evolution under one antibiotic (X)

Therapy using collateral sensitivity
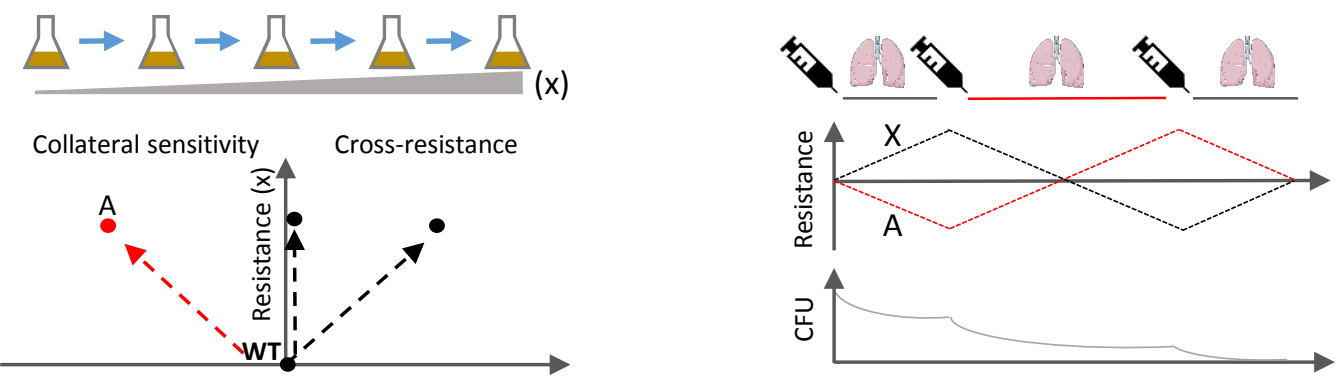

Resistance for other antibiotics

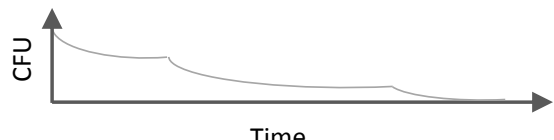

C

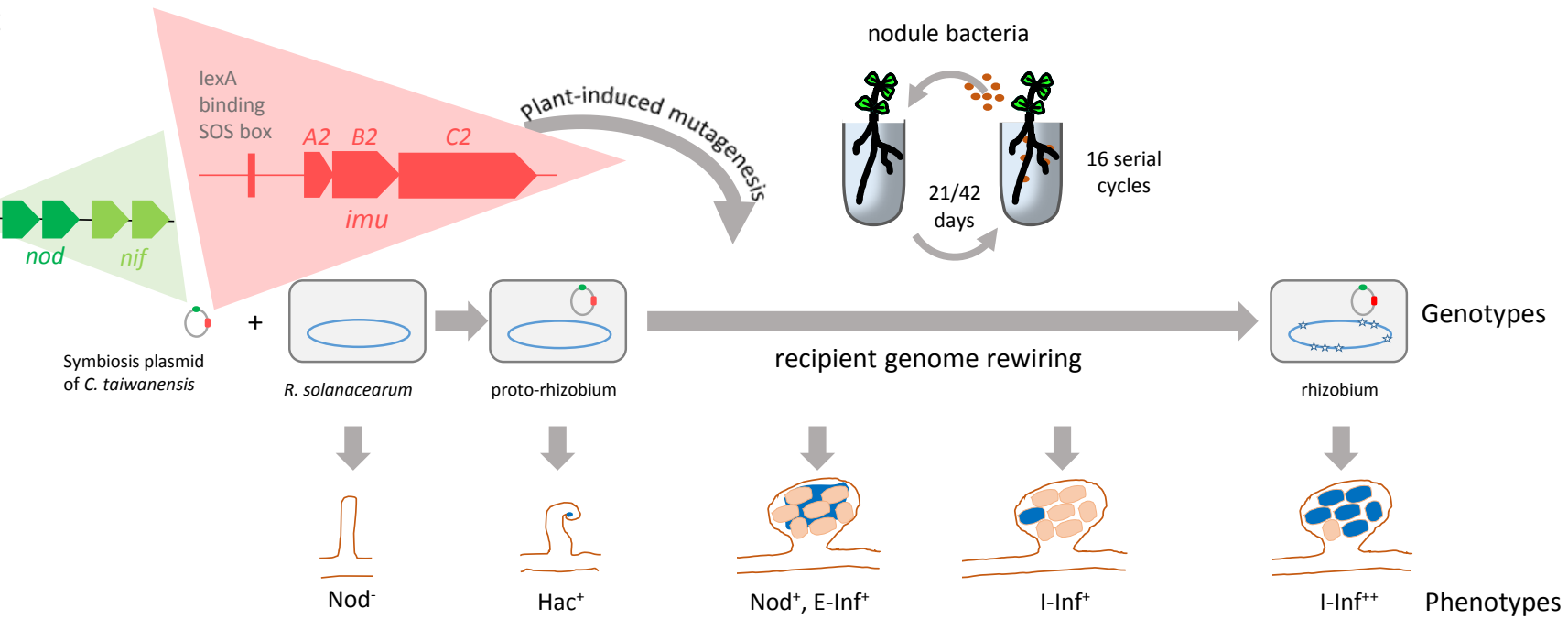




\section{Ancestor}

\section{Complexity}

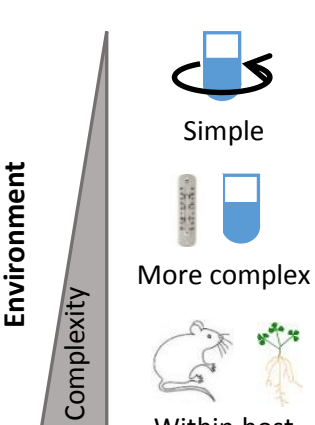

\begin{tabular}{|l|}
\multicolumn{1}{c}{ Clone } \\
\hline $\begin{array}{l}\text { Escherichia coli/minimal medium in batch } \\
\text { cultures [89] or chemostat [90] }\end{array}$ \\
\hline \\
\hline $\begin{array}{l}\text { Escherichia coli/antibiotics [37, 50, 96] } \\
\text { Pseudomonas fluorescens /static microcosms } \\
\text { [91] }\end{array}$ \\
\hline
\end{tabular}

Genetically modified

Escherichia coli/minimal or rich medium $[19,24,84,85]$

Pseudomonas fluorescens/ spreading motility [17]

Myxococcus xanthus/ swarming [107]

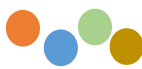

Population/community

Desulfovibrio vulgaris/Methanococcus maripaludis [86]

Acinetobacter/Pseudomonas putida [87]

Desulfovibrio vulgaris/Methanococcus maripaludis [86]

Acinetobacter/Pseudomonas putida [87]
Escherichia coli/mouse gut [99] Vibrio fischeri /squid [98]

Pseudomonas aeruginosa / worm [97]

Ralstonia solanacearum/ host plants [57]
Ralstonia solanacearum /legumes [66] Escherichia coli/mouse gut [99]
Staphylococcus aureus-Enterococcus

faecalis /worm [72]

Escherichia coli/mouse gut [88] 


\section{Highlights}

- Experimental evolution (EE) can complement traditional molecular genetic studies on microbial systems.

- The diversity of EE approaches enabled progresses in many fields of microbiology, including molecular mechanisms of gene regulation, antibiotic resistance, and hostmicrobiome interactions.

- Under specific conditions, EE can parallel the evolution of natural systems.

- EE offers exciting perspectives to discover the function of new genes and probe evolution within communities. 


\section{Outstanding questions:}

- Can we exploit mutations found in EE to characterise genes of unknown function?

- Can EE enlighten mechanisms slowing down the evolution of resistances?

- Can the EE of microbial communities bring new insights into their functional characteristics?

- Can we use within-host EE to identify host factors (and other environmental factors) shaping bacterial evolutionary trajectories during infection?

- Can EE reproduce the emergence of major intracellular symbiotic associations (such as mitochondria in eukaryotic cells, plastids in the green lineage, association between fungi and land plants, obligate endosymbionts of insects)? 